\title{
La canción protesta y los discursos de contracultura y resistencia durante la década de los sesenta en Colombia*
}

\author{
Protest song and countercultural \\ discourses of resistance in 1960s Colombia
}

\author{
Joshua Katz-Rosene** \\ Franklin \& Marshall College, Estados Unidos
}

Traducción: Daniel Castelblanco

DOI: $10.22380 / 2539472 X .2015$

\begin{abstract}
RESUMEN
Este ensayo examina tres movimientos contraculturales que cautivaron a un amplio sector de la juventud en las principales ciudades de Colombia durante la década de los sesenta: el movimiento de la canción protesta, la subcultura del rock and roll, cuya identidad local se denominó nueva ola, y el nadaísmo, un movimiento literario de vanguardia. Analizo las correspondencias y discontinuidades entre las formas en que los participantes de estos movimientos concibieron los medios ideales para llevar a cabo la resistencia social, cultural y política. Sostengo que, si bien hubo tensiones fundamentales entre los "discursos de resistencia" asociados a cada una de estas corrientes contraculturales, su convergencia a finales de la década de los sesenta facilitó el surgimiento de una variante comercial de canción protesta.
\end{abstract}

Palabras clave: canción protesta, nadaísmo, nueva ola, resistencia.

\begin{abstract}
This essay examines three countercultural-oppositional movements that captivated a wide swath of youth in Colombia's biggest cities during the 1960s: the canción protesta (protest song) movement, the rock and roll subculture denominated as nueva ola (new wave), and nadaísmo, a rabblerousing avantgarde literary movement. I analyze the correspondences and discontinuities in the ways adherents of these movements conceived of the ideal means to carry out social, cultural, and political resistance. While there were fundamental tensions between the "discourses of resistance" linked to these three countercultural streams, I argue that their convergence in the late 1960s facilitated the emergence of a commercial form of canción protesta.
\end{abstract}

Keywords: protest song, nadaísmo, new wave, rock and roll, resistance.

El autor agradece a Gloria Bongcam, Pablus Gallinazo, Mario Osorio Marín, Norman Smith, Ana Valencia, Ricardo Waldmann y muchos otros por compartir sus recuerdos de los acontecimientos discutidos en este artículo. Gracias a Daniel Castelblanco por su magnífica traducción, a una subvención de Franklin \& Marshall College por el soporte económico y al equipo de la RCA. Este artículo fue originalmente publicado en inglés en la revista Resonancias (Pontificia Universidad Católica de Chile) 24, n. ${ }^{\circ}$ 47, https://doi.org/10.7764/res.2020.47.3

**_josh@telepath.ca / https://orcid.org/0000-0003-3736-007X 


\section{Introducción}

El 16 de julio de 1971 se inauguró el Segundo Festival de la Canción Protesta Coco de Oro en medio de una controversia en San Andrés, territorio insular de Colombia en el mar Caribe. El principal descontento que expresaron sus participantes giraba en torno a la composición del jurado que presidiría el evento central del festival: el concurso por la mejor canción protesta. Haciendo eco del rechazo generalizado hacia la presencia de funcionarios del Gobierno en el jurado, un columnista preguntó deliberadamente:

¿Qué diablos tiene que hacer en un festival de protesta el ministro de Gobierno? ¿Cómo se puede asegurar imparcialidad en el dictamen si un defensor oficioso y oficial del establecimiento está encargado de dar su voto sobre las canciones? [...] Porque entre los jurados hay, no uno, sino varios, delegados de un gobierno que no es propiamente revolucionario. Ellos tendrán buen cuidado de que las canciones elegidas no sean "muy fuertes" y de que el estado de sitio se extienda a las obras presentadas. (“Contenido" 1971, 18)

El periodista aludía al Estatuto de Seguridad promulgado a principios de 1971 por el gobierno conservador de Misael Pastrana en respuesta a las crecientes protestas estudiantiles, sindicales y campesinas, mediante el cual fueron prohibidas aquellas reuniones de orientación política que pudieran "perturbar la paz" (citado en Archila Neira 2003, 105). El periodista pudo haberse preguntado: ¿cómo serían apreciadas algunas de las canciones inscritas en el concurso - como Destino la guerrilla - si los miembros del jurado invocaban las restricciones propias del estado de sitio? Esta canción ensalza a las guerrillas marxistas que entonces estaban en guerra con el Estado colombiano:

Caminando, caminando

La guerrilla es un amor

Echan pata que da miedo

Viva la revolución

Pese a las curiosas invitaciones a representantes del Gobierno para servir como jueces, la composición general del jurado estuvo en consonancia con el carácter multidimensional del festival. Varias figuras destacadas en el ámbito cultural a nivel nacional integraron la mesa de jurados: Santiago García, director de la Casa de la Cultura — identificada con el Partido Comunista - en el centro de Bogotá; el poeta Gonzalo Arango, fundador del movimiento nadaísta; el locutor y productor Alfonso Lizarazo; el empresario local Nicolás Jackaman; y periodistas del diario El Tiempo, entre otros. 
La oferta musical del festival también fue variada. Entre los cantantes de música protesta más representativos de Colombia estuvieron Pablus Gallinazo, compositor de Destino la guerrilla, y el dúo Ana y Jaime. Ambos grupos habían estado involucrados en el reciente florecimiento de la canción protesta en la Casa de la Cultura de Bogotá, pero también estaban trabajando con Alfonso Lizarazo y la industria mediática que se había construido en torno a la escena rockera de la nueva ola para promover su música. Las invitaciones que ciertos artistas recibieron para actuar en el festival, como la estrella Harold y la banda de rock Los Flippers, acentuaron los lazos que existían entre algunos practicantes de la canción protesta y la nueva ola durante este periodo. Aunque la presencia de figuras nuevaoleras estuvo en consonancia con la tendencia generalizada, también fueron invitados cantantes de pop que no tenían una relación previa con la canción protesta (“II Festival de la Canción” 1971).

Mientras el jurado determinaba qué clase de canciones recibiría los premios más destacados del concurso de canción protesta, los periodistas que cubrían el evento sintieron que las composiciones interpretadas no estaban a la altura del festival. Un periodista con el seudónimo de Samuel (1971a) lamentó que pocos participantes trabajaran seriamente en el campo de la canción protesta. En su opinión, Ana y Jaime - quienes obtuvieron el tercer lugar con una canción titulada Obreros - deberían haber ganado el primer premio en razón a que su principal línea de trabajo artístico había sido como cantantes de protesta. Su valoración general del festival se resumió en el título que dio a uno de sus artículos: "Festival de San Andrés más canción que protesta” (Samuel 1971b)ํ. La periodista de El Tiempo Alegre Levy, quien formó parte del jurado, ratificó muchas de las preocupaciones de Samuel. Ella se quejó de la reunión aleatoria de artistas que cantaban canciones "que tenían de todo menos contenido de protesta”, mas reconoció que el artista venezolano Manuel de la Roche "trajo una canción verdaderamente de protesta titulada “Café y petróleo”' (Levy 1971, 17). Levy esperaba que las ediciones futuras del festival fueran más consistentes con lo que ella veía como el espíritu de la canción protesta: "Deseamos que el próximo año haya más protesta, más pueblo y menos establecimiento” (Levy 1971, 17).

Al final, el Coco de Oro a la mejor canción protesta fue para el intérprete bogotano Sergio Torres, por su cumbia No trabajo más. A pesar de que Torres no estaba vinculado al movimiento de la canción protesta, su letra era consistente con gran parte del repertorio de la música protesta de la época, pues presentaba una denuncia marxista más bien superficial contra la explotación capitalista:

1 También advirtió que el costo de la entrada (50 pesos) excluía "[al] pueblo, quien es al que debe ir dirigido el mensaje" (Samuel 1971b, 19). 
Coro

No trabajo más

Pa ningún patrón

Solo espero ya

La Revolución

[...]

Trabajando arando la tierra

Pa sembrar el algodón

Mientras las utilidades

Las disfruta mi patrón.

En un desconcertante final, el trofeo a la mejor canción protesta fue otorgado por el embajador de Guatemala, un país gobernado por un coronel de mano dura que perseguía a la oposición de izquierda sin piedad ("Entrega del 'Coco de Oro’” 1971).

La extensa cobertura periodística del Festival Coco de Oro de 1971 nos proporciona una amplia visión de los itinerarios de la canción protesta solo algunos años después de que esta categoría se estableciera en Colombia. Al expresar sus convicciones sobre qué tipo de artistas y canciones debían participar en un festival de ese género, qué personas debían ser sus jurados y qué público era el más apropiado para recibir sus mensajes, aquellas personas que se mostraron decepcionadas por la escasez de legítima protesta en el evento estaban articulando los principios de lo que habría de convertirse en el núcleo discursivo de la canción protesta. No obstante, el simple hecho de verse obligadas a expresar sus posturas demuestra que enfrentaban percepciones antagónicas sobre aquello que constituye el género. De hecho, los comentarios acalorados que suscitó la diversidad de organizadores, artistas, jueces y asistentes al festival son testimonio del choque que existía en ese momento entre varios discursos de la cultura popular colombiana.

En este artículo examino tres corrientes contraculturales que cautivaron a gran parte de la juventud en las ciudades más grandes de Colombia durante la década de los sesenta: el nadaísmo, la nueva ola y la canción protesta. Después de describir su desarrollo, analizo las similitudes y diferencias entre las formas en que los adeptos de dichos movimientos concibieron los medios ideales para efectuar cambios culturales y políticos. Evidentemente, entre quienes se encontraban en el núcleo de estos movimientos existieron tensiones fundamentales alrededor de sus diversas posturas sobre cómo hacer resistencia. Los activistas 
comunistas que inicialmente cultivaron la canción protesta fueron particularmente desdeñosos de las que percibían como artificiosas tácticas revolucionarias asociadas a otros campos contraculturales. Sin embargo, es claro que hubo interacción y admiración recíproca entre los exponentes de distintas corrientes; la realidad para la mayoría de los jóvenes urbanos era la de un entorno contracultural multidimensional que ofrecía una variedad de posibilidades para la expresión artística y la acción política.

El Festival Coco de Oro y la atención que le otorgaron los medios de comunicación convencionales fueron también síntomas de la transición en que se encontraba la canción protesta a principios de la década de los setenta, desde un movimiento de base a un fenómeno comercialmente viable. En última instancia, sostengo que el paso de la canción protesta al ámbito comercial fue acelerado por la confluencia de los discursos de resistencia vinculados a las tres corrientes contraculturales dominantes a finales de la década de los sesenta.

\section{Discursos de resistencia}

No está de moda escribir sobre música y resistencia en 2020. Hablando en 2014 sobre su libro Sigue adelante: raza, poder y música en Nueva Orleans ${ }^{2}$, el etnomusicólogo Matt Sakakeeny opinó que dicho enfoque analítico era anacrónico: “¿Te imaginas [preguntó a su entrevistador Steven Feld] si hubiera organizado mi estudio en torno a estructura/agencia, resistencia/acomodación o hegemonía/ complicidad hoy?” (Dilday 2014).

El argumento de Sakakeeny es bien recibido. La proliferación de investigaciones sobre resistencia en las ciencias sociales a partir de la década de los ochenta ha generado abundantes críticas sobre el empleo inconsistente del término. Las sociólogas Jocelyn Hollander y Rachel Einwohner (2004) hicieron un inventario preliminar de los principales problemas que identificaron en la literatura, los cuales giraban en torno a dos cuestiones: si los actos de resistencia deben ser reconocibles para quienes están dirigidos y sus observadores y si detrás de ellos debe haber una intención de resistencia. En su reciente libro Conceptualizing 'everyday resistance' (Conceptualizando la 'resistencia cotidiana'), Anna Johansson y Stellan Vinthagen (2020) proporcionan una revisión completa de las

2 La versión original del libro fue publicada en 2013 bajo el título Roll with it: brass bands in the streets of New Orleans. 
trampas teóricas que encuentran en la producción académica sobre la resistencia cotidiana; aun así, la publicación de este volumen demuestra un continuo interés académico en el estudio de la resistencia.

El título del libro de Johansson y Vinthagen indica el hecho de que la mayoría de la literatura académica sobre resistencia en realidad se basa en la investigación seminal de James Scott (1985) sobre resistencia cotidiana. Scott describió esta última como conformada por acciones descoordinadas y a menudo irreconocibles, y se propuso teorizarla en respuesta a las investigaciones que se enfocaban de manera desproporcionada en el estudio de formas de resistencia coordinadas y explícitas. Pese a sus esfuerzos por diferenciar estos fenómenos, la idea de resistencia cotidiana desarrollada por Scott ha sido amalgamada en gran parte con la noción académica de resistencia en su forma indiferenciada. Esta fusión es evidente en muchos de los escritos sobre música. En su entrada sobre "Resistencia y protesta" en la Continuum encyclopedia of popular music of the world (Enciclopedia continuum de música popular del mundo), por ejemplo, Dave Laing $(2003,345)$ estableció una diferencia entre canciones de protesta, a las que definió como “declaraciones de oposición explícitas”, y la "expresión de disidencia [...] codificada u opaca” que se encuentra en la música de resistencia. Esta definición ha sido replicada en numerosos estudios (Attfield 2017; Laine, Suurpää y Ltifi 2018; Tranmer 2019), incluyendo el libro frecuentemente citado de John Street (2012) Music and politics (Música y política).

No hay duda de que debemos prestar atención a las diferentes formas en que opera la resistencia musical en los espectros entre la oposición velada y la protesta explícita, y entre la lucha a pequeña y a gran escala. No obstante, distinguir las canciones de protesta de la música de resistencia tan solo invierte un problema que Scott $(1985,297)$ trató de solucionar en su trabajo: la tendencia a “reservar el término 'resistencia' para la acción colectiva u organizada”. Si seguimos el enunciado de Laing, la resistencia musical se restringe a la expresión individual en la que los mensajes de oposición se encuentran ocultos, mientras que los cantantes de protesta hacen algo intrínsecamente distinto. Concuerdo con Moreno Almeida $(2017,3)$ en cuanto a que esta suposición es subjetiva.

Otro dilema se pone de manifiesto cuando reconocemos que los esfuerzos académicos por analizar la resistencia generalmente apuntan a las teorías de la resistencia cotidiana. Si, por definición, la resistencia cotidiana no tiene “ninguna conspiración leninista detrás” (Scott 1985, 31), entonces los modelos empleados para estudiarla pueden ser de limitada utilidad para investigar un repertorio musical que fue fuertemente informado por ideas marxistas-leninistas, como ocurrió en el caso de la canción protesta colombiana. 
En vista de los desafíos que conlleva examinar la música de protesta a través del prisma de la resistencia, ¿por qué convertirla en un tema central de este estudio? Por un lado, porque es imperativo prestar atención a la terminología utilizada en las comunidades con las que trabajamos (Gledhill 2012, 1-2); por otro, porque la investigación sobre la resistencia cotidiana ha dado lugar a conocimientos valiosos que nos ayudan a pensar en otras formas de resistencia y acción política.

Entiendo los movimientos contraculturales discutidos en este artículo como estrechamente emparentados con discursos de resistencia. Permítanme ilustrar mediante el ejemplo de la canción protesta - una categoría a través de la cual un subconjunto de músicos y activistas en la Colombia de la década de los sesenta se esforzó por definir cómo debería ser y sonar la resistencia musical-. La mayoría de las personas involucradas en la canción protesta eran estudiantes de educación superior o personas relacionadas con organizaciones políticas de izquierda. Inspirados en los textos marxistas que devoraban, los participantes del movimiento posicionaron la canción protesta como una forma de lucha contra el sistema capitalista impuesto por la oligarquía colombiana, que sostuvo su dominación política a través de una democracia severamente restringida. Los ideólogos de izquierda invocaron ad nauseum el concepto de lucha para denotar las diferentes modalidades de resistencia a través de las cuales buscaban lograr sus objetivos. A principios de la década de los sesenta, por ejemplo, el Partido Comunista de Colombia había concretado una estrategia conocida como "la combinación de todas las formas de lucha”, mediante la que proponía impulsar su agenda a través de la política electoral y la resistencia armada, junto con huelgas, invasiones de tierras, etc. Los comentaristas debatieron enérgicamente el papel que el arte debía asumir en este marco - en otras palabras, su potencial de resistencia-. Como describo a continuación, los participantes de movimientos como el nadaísmo y la nueva ola elaboraron sus propios discursos de resistencia en conversación con un conjunto de recursos ideológicos diferentes.

Hasta ahora, he hecho énfasis en el rol de quienes participan en actos de resistencia al orientar el discurso sobre esta. Sin embargo, reconozco que la resistencia - cualquiera que sea su tipo- es "articulada discursivamente por actores, objetivos y observadores [...] debido a su entrelazamiento y relaciones interseccionales con el poder" (Vinthagen y Johansson 2013, 39). Este argumento condensa tres presunciones interrelacionadas que deseo resaltar a propósito de la manera en que concibo los discursos de resistencia. En primer lugar, amplía de forma apropiada la red de actores dentro de la cual vemos la resistencia en acción y nos obliga a prestar mucha atención a las reacciones que puede provocar —o a la falta de ellas-. En segundo lugar, reconoce los enfoques que muchos académicos 
han propuesto (Abu-Lughod 1990; Gledhill 2012; Moreno Almeida 2017) —inspirados por Foucault (1978) - en torno a la relación infinitamente compleja entre resistencia y poder. Finalmente, el postulado de Vinthagen y Johansson habla de la importancia de cuestionar "las tácticas discursivas que imbuyen los actos de resistencia con sus significados particulares” (McDonald 2013, 28), en contextos históricos y sociales específicos (Johansson y Vinthagen 2020, 6).

En las páginas siguientes me enfoco en los contextos que forman el telón de fondo de mi investigación sobre música, protesta y resistencia en Colombia.

\section{La Violencia, el Frente Nacional y la primera generación de guerrillas}

Desde mediados del siglo XX, los colombianos han estado lidiando con las consecuencias que dejó el periodo de más de diez años de violencia política sectaria que se conoce como La Violencia. Su inicio suele estar vinculado al 9 de abril de 1948, fecha en que fue asesinado en Bogotá el líder del Partido Liberal, Jorge Eliécer Gaitán, cuya retórica de tintes socialistas le había traído enorme popularidad. Este hecho desató el Bogotazo, varios días de disturbios masivos en la capital que se extendieron a otras localidades y se tradujeron en incesantes enfrentamientos entre simpatizantes de los principales partidos políticos del país, el Liberal y el Conservador. Irónicamente, fue necesario un golpe del general Gustavo Rojas Pinilla en 1953 para dar un respiro a la violencia. Sin embargo, a medida que avanzaba la dictadura, los niveles de violencia resurgieron y los partidos oficiales pronto desconfiaron de los esfuerzos de Rojas por permanecer en el poder.

En 1957 los votantes aprobaron un plebiscito que autorizó la creación del Frente Nacional (FN), un sistema que garantizaba la representación equitativa de liberales y conservadores en los órganos legislativos y la alternancia de la presidencia entre los partidos durante los siguientes dieciséis años. Sin duda, el pacto allanó el camino para terminar con La Violencia en mayúscula, pero el acuerdo bipartidista marginó a las fuerzas políticas alternativas, situación agravada por el contexto generalizado de la Guerra Fría. Con el triunfo de la Revolución cubana, tan solo un año después de la creación del FN en 1958, la contención del "fantasma comunista” se convirtió en una prioridad para los sucesivos presidentes del acuerdo (Archila 2003, 93). De hecho, desde mediados de la década de los sesenta, las primeras líneas de combate en el conflicto colombiano se volvieron a trazar entre el ejército colombiano y un puñado de guerrillas recién fusionadas. 
Las teorías revolucionarias del "Che” Guevara influyeron en la creación de algunos de los primeros grupos guerrilleros comunistas en Colombia. El Ejército de Liberación Nacional (ELN), por ejemplo, fue fundado en 1964 por radicales de clase media que recibieron entrenamiento militar en Cuba. Las Fuerzas Armadas Revolucionarias de Colombia (FARC), por otro lado, surgieron en 1966 a partir de la unión de una multitud de grupos de autodefensa campesina que se habían formado bajo la influencia del Partido Comunista durante La Violencia. $\mathrm{Al}$ año siguiente se creó el Ejército Popular de Liberación (EPL) como la rama armada del Partido Comunista Marxista-Leninista, una facción maoísta que se separó del Partido Comunista de Colombia (PCC).

Uno de los sectores entre los que la guerrilla reclutó nuevos combatientes fue el movimiento estudiantil universitario. Una expresión común entre los organizadores estudiantiles durante este periodo fue “irse pa'l monte”, que evoca las remotas zonas montañosas donde se refugiaban las guerrillas (Beltrán 2002, 169). Los relatos de las actividades del movimiento estudiantil durante la década de los sesenta muestran un ciclo continuo de protestas que fueron enfrentadas por las autoridades con el cierre de universidades, así como con su ocupación policial y militar. Estas respuestas represivas lanzaron a los líderes estudiantiles a los brazos abiertos de las alas juveniles de los partidos de izquierda y, a partir de entonces, la organización estudiantil estuvo dominada por la Juventud Comunista (JUCO) y los grupos izquierdistas rivales (Archila 2003).

\section{Nadaísmo}

El nadaísmo [...] más que una manifestación tardía de nuestro débil vanguardismo estético, fue la protesta de una intelectualidad de orígenes más o menos plebeyos contra la oficialidad cultural que había dado licencia moral de funcionamiento a los mecanismos de la violencia política. [...] La violencia política había incitado, pues, la existencia de los desplantes nadaístas en una sociedad que sacralizó con bendiciones, rezos y camándulas los rituales de mutilación, desollamiento e incineración que se repetían sin piedad. (Loaiza 2004, 86)

Como deja en claro el historiador Gilberto Loaiza, el movimiento literario nadaísta que surgió en 1958 fue en gran medida una respuesta a la violencia que azotó a Colombia durante la década previa. De hecho, el impulso inicial del movimiento estuvo ligado a acontecimientos políticos que marcaron la disminución 
de La Violencia y el inicio del Frente Nacional. En 1957 Gonzalo Arango, fundador del nadaísmo, había trabajado como delegado suplente en la Asamblea Nacional Constituyente que tenía la intención de extender el régimen de Gustavo Rojas Pinilla. Cuando el general renunció ese año en medio de una creciente oposición, Arango huyó a Cali para refugiarse de la reacción violenta contra los partidarios rojistas en la ciudad de Medellín (Romero 1988, 35). Una vez en Cali, redactó el Primer manifiesto nadaísta que publicó al regresar a Medellín en 1958. En este texto fundacional, propuso que el nadaísmo era "un estado del espíritu revolucionario" que pretendía desacreditar todas las facetas del "orden establecido” y destacó el dominio poético como el frente en el que se libraría la batalla con la sociedad colombiana (Arango 2013).

En sus esfuerzos por socavar todas las facetas de la tradición nacional, los nadaístas exhibieron un “cosmopolitismo feroz” (Romero 1988, 39) y construyeron su postura inconformista bajo la guía de luminarias literarias y filosóficas extranjeras (Pedersen 1971, 355). Sus influencias fundamentales incluyeron el existencialismo de Jean-Paul Sartre (Lagos 1977, 104) y el movimiento surrealista (Romero 1988, 77-78). Desde principios de los sesenta, diversas corrientes de la contracultura estadounidense también ejercieron un impacto creciente. Cuando el poeta Elmo Valencia se unió a los nadaístas de Cali en 1960 tras concluir sus estudios en Estados Unidos, aportó sus conocimientos sobre los beatniks estadounidenses. A finales de la década, la lucha de los nadaístas por romper las convenciones en torno a la vestimenta, la longitud del cabello y las relaciones sexuales encontró resonancia en la cultura hippie euroamericana. Los nadaístas más prominentes defendieron a los emisarios de los hippies que aparecían a través de los medios masivos de comunicación (por ejemplo, Los Beatles y Bob Dylan) y muchos de ellos experimentaron con drogas.

Si bien los nadaístas eran ambivalentes frente a la política organizada, la cita que introduce esta sección alude a una institución que fue el blanco inequívoco de su ira: la Iglesia católica. Conocida por sus tendencias conservadoras y vínculos con los partidos políticos dominantes, la Iglesia colombiana fue acusada incluso de fomentar el derramamiento de sangre durante La Violencia. En las décadas previas, su control sobre la vida social y política en Medellín fue omnipresente (Tirado 2014, 212). Los nadaístas se propusieron atacar este símbolo del status quo desde un principio. En 1959 un grupo de ellos interrumpió la misa en la basílica de Medellín, destrozó las hostias de la comunión y fumó cigarrillos antes de ser perseguidos por una turba enfurecida (Romero 1988, 40-41). 


\section{Nueva ola}

Durante los mismos años en que los intelectuales de Medellín y Cali sentaban las bases del nadaísmo, el rock and roll llegó a Bogotá y generó una subcultura vigorosa. A finales de los años cincuenta, el locutor Carlos Pinzón se convirtió en el promotor más destacado del rock and roll norteamericano a través de su programa en la emisora radial Nuevo Mundo (Pérez 2007, 27-29; Riaño 2014). Sembrando las semillas para el desarrollo de una escena local de rock en español, esta y otras estaciones de la capital pronto comenzaron a transmitir las versiones de ciertos éxitos del rock and roll estadounidense grabadas en español por bandas mexicanas y argentinas. Entre las primeras agrupaciones formadas en Colombia a principios de la década de los sesenta estuvieron Los Speakers y Los Flippers, que impulsaron el desarrollo del rock nacional al componer canciones originales.

El término nueva ola fue acuñado en Argentina al concluir la década de los sesenta para denotar los estilos de rock and roll extranjeros que se estaban infiltrando en las ondas radiales, junto con sus exponentes locales (Manzano 2010, 19, 35). Puesto que estos artistas ocuparon un lugar destacado en las listas de las primeras estaciones radiales que dedicaron parte sustancial de su programación al rock and roll en Colombia, la expresión nueva ola pasó a usarse de forma genérica para identificar toda la música derivada del rock que estas transmitían (Riaño 2014). Aprovechando la creciente popularidad del género, en 1963 Carlos Pinzón inició un nuevo canal enfocado en la nueva ola para la cadena Caracol. Radio 15, como se bautizó la emisora, pasó a manos de Alfonso Lizarazo, quien también heredó un programa llamado El show de los frenéticos, que semanalmente transmitía un concurso de talentos en vivo. En 1965 Radio Cordillera estrenó un espectáculo similar llamado El club del clan y al año siguiente se emitió una adaptación para la televisión. Programas como estos se convirtieron en el principal medio a través del cual los músicos de la nueva ola lanzaron sus carreras.

Hasta alrededor de 1968 se habían hecho pocos esfuerzos por establecer distinciones clasificatorias entre los diferentes tipos de artistas agrupados en la categoría de música juvenil que tomaba al país por asalto. Aunque con frecuencia era etiquetado como nueva ola, el rock and roll hecho en casa a mediados de la década de los sesenta también se conocía en Colombia como go-gó y ye-yé, términos supuestamente derivados de las exclamaciones cantadas con frecuencia en el rock and roll en inglés (Riaño 2014). Hacia 1967-1968, sin embargo, los sonidos más mesurados de la nueva ola —o go-gó/ye-yé-comenzaron a distinguirse de la orientación ideológicamente más inconformista y musicalmente progresiva 
de los artistas de rock que intentaban mantenerse al día con las tendencias internacionales. Como escribe Umberto Pérez (2007):

Es importante aclarar que para entonces la nueva ola ya se diferenciaba del rock. A ella pertenecían sobre todo los cantantes solistas y la gente de “El club del clan”, las canciones eran poco rebeldes o contestatarias, se encontraban alejadas de los ritmos acelerados del rock y se acomodaban más fácilmente a los oídos de los adultos. (65)

De hecho, como veremos, en el curso de unos pocos años la línea que separaba al rock de una categoría musical que se definía por su postura contestataria -la canción protesta- se volvió algo borrosa.

\section{Canción protesta}

Mientras el nadaísmo se aproximaba a su décimo aniversario y la nueva ola alcanzaba su auge, otro movimiento cultural de orientación juvenil tomaba forma entre los círculos de izquierda. Hacia 1966-1967 un pequeño grupo de músicos comenzó a actuar en sindicatos, universidades y espacios comunitarios en Bogotá en conjunto con el PCC y la JUCO. Estos fueron los primeros artistas colombianos en ser identificados con la etiqueta de canción protesta. En noviembre de 1967, un reportaje sobre la celebración del cincuentenario de la Revolución rusa, publicado en el semanario del PCC, Voz Proletaria, afirmaba que Pablus Gallinazo, quien actuó en el evento, era "tal vez el mejor exponente de la nueva tendencia bautizada como la canción protesta” (“Grandioso acto” 1967). Varios meses después, un grupo de cantantes que incluía a Aída Pérez, Juan Sebastián y Eliana ofreció un "recital de canciones de protesta” en una exposición de dibujos vietnamitas en la Casa de la Cultura (“Noticiero cultural” 1968).

La idea de cultivar una categoría musical distinta denominada canción protesta parece haber venido de Cuba. Allí se llevó a cabo en 1967 el Encuentro Internacional de la Canción Protesta y, si bien Colombia no estuvo representada en el evento, es probable que aquellos activistas del PCC que habían establecido vínculos con Cuba transmitieran noticias desde la isla. Músicos activistas como Alejandro Gómez Roa, quien había actuado frente a Fidel Castro en La Habana en 1960 (Emanuelsson 2014), habrían sido conscientes de que dicho encuentro condujo a la creación del Centro de la Canción de Protesta en la Casa de las Américas de La Habana (Díaz 1994, 126-133). Gómez y sus compañeros se inspiraron para formalizar su propio trabajo de manera semejante. 
El 24 de abril de 1968 se inauguró el Centro Nacional de la Canción Protesta (CNCP) en la Casa de la Cultura de Bogotá, con el objetivo de crear un "movimiento que aglutine por medio de las canciones a un vasto sector de la juventud inconforme” (“Canciones de protesta y esperanza” 1968). Pese al aire institucional que evoca su nombre, el conjunto variable de músicos involucrados en el CNCP inicialmente continuó haciendo su trabajo de la misma forma en que lo había hecho durante los años previos a su fundación. Si bien daban recitales en el centro mismo, siguieron siendo convocados para amenizar eventos organizados por la JUCO.

Durante el periodo comprendido entre 1968 y 1969 se gestaron varias de las iniciativas que el CNCP lanzó a partir de 1970. Ese año, comenzó a albergar peñas semanales para "poetas, cantantes de baladas, canción protesta y música folclórica” (“Programación” 1970). Las peñas se inspiraron en la ahora famosa Peña de los Parra (Gómez 1973), fundada en Santiago de Chile en 1965 por los cantantes de nueva canción Isabel y Ángel Parra. En 1968 el dramaturgo chileno Gustavo Gac y la actriz colombiana Perla Valencia — quienes habían realizado una gira llevando un espectáculo de música y poesía desde Chile hasta Colombia- se establecieron como miembros integrales de la Casa de la Cultura y transmitieron su conocimiento sobre el ambiente de la peña chilena en el que la nueva canción prosperaba (Flores 1994, 145).

En septiembre de 1970 Gac, Valencia y otros organizaron el Primer Festival de la Canción Protesta, un evento de una semana de duración auspiciado por el CNCP. Con la clara intención de difundir entre un público más amplio el trabajo político-artístico que venían cultivando, los coordinadores invitaron a músicos de varios países de Latinoamérica, programaron conciertos en sedes sindicales, universidades y barrios populares, y trataron de conseguir que fueran emitidos por radio y televisión. Al menos una docena de artistas participaron (“Organizan festival” 1970).

Un periodo de intensa actividad continuó en el CNCP hasta principios de 1971, cuando el Centro se asoció con ciertos sindicatos para reformular las peñas semanales como Sábados Obreros. El objetivo de esta serie de presentaciones fue generar interés en la retórica marxista de parte del sujeto social que los músicos del CNCP evocaban con frecuencia en los textos de sus canciones: el obrero. Si bien el costo de entrada a las peñas era de diez pesos (Gac y Valencia 1970), a los trabajadores afiliados al sindicato se les ofreció la entrada a los Sábados Obreros por solo un peso (“Los sábados obreros” 1971). 


\section{Discursos divergentes}

Las diferencias entre las tres expresiones (izquierda, nadaísmo y rock) eran enormes y obvias. Mientras los dos primeros poseían algún grado de organización e ideología, el rock aparecía como un fenómeno amorfo y confuso. [...] Sin embargo, solo el nadaísmo comprendió el significado de esta manifestación juvenil y de alguna manera se vinculó a ella mediante lazos de amistad y pequeñas contribuciones literarias. [...] Los comunistas y otros grupos revolucionarios no entendieron el fenómeno y lo ubicaron en los casilleros tradicionales de penetración imperialista y propuesta alienante. [...] Y es que para la época el mensaje hippie de paz y amor no encajaba con la reciente tragedia de Camilo Torres y el torrente de revolución armada que recorría el país. (Giraldo 1997, 15)

Como observa Jorge Giraldo, había límites claros y, en ocasiones, infranqueables que separaban las formas en que los adeptos del nadaísmo, la nueva ola, el rock hippie y la canción protesta concebían la resistencia a la cultura dominante y al sistema político-económico. En este apartado analizo las múltiples discontinuidades entre los discursos de resistencia fomentados en torno a estas corrientes contraculturales. En la siguiente sección identifico las convergencias entre los artistas, las audiencias y los contextos de intervención artística asociados con cada uno durante los años finales de la década de los sesenta.

\section{La política de la nada}

Aunque el repudio de los nadaístas a la cultura impuesta por las élites de los partidos políticos tradicionales y la Iglesia católica coincidió con ciertas posturas de la izquierda, su relación con la izquierda política fue tensa. Como escribió el poeta nadaísta Eduardo Escobar (1976): "El nadaísmo nada tiene que decir en el conflicto del capitalismo y el comunismo. [...] No va dirigido a las masas obreras ni campesinas” (8). No hace falta decir que aquí hay una discrepancia irreconciliable con la premisa fundamental del marxismo, una ideología que los grupos comunistas buscaban comunicar sobre todo a los trabajadores y campesinos. En una valoración del nadaísmo con motivo de su vigésimo aniversario, Escobar afirmó: "Nuestras relaciones con la izquierda han sido, desde la complicidad en el trabajo hasta nuestra indiferencia y su franco rechazo" ("Lo que dejó la tempestad” 1979). Sin embargo, continuó enfatizando que los nadaístas habían sido categóricos en cuestiones políticas clave, como su apoyo inquebrantable a la Revolución cubana. 
Dada su irreverencia hacia las instituciones y figuras intelectuales y políticas de todo tinte, no es de extrañar que los nadaístas mismos fueran objeto de mucha crítica. Si bien la condena del clero y las élites era predecible, aquellos izquierdistas que podrían haber guardado cierta simpatía por el nadaísmo en virtud de su actitud antisistema criticaron su enfoque escandaloso por considerar que no se apartaba verdaderamente de los valores burgueses ("Del nadaísmo al oficialismo” 1968). Escobar rebatió estos puntos de vista argumentando que la izquierda no podía comprender la proposición revolucionaria del nadaísmo y su “afirmación del cuerpo, de la vida, la tierra, el amor y la negación de absolutos” ("Lo que dejó la tempestad” 1979, 27).

Como señaló Giraldo Ramírez en la cita anterior, hubo muchos puntos de intersección entre el nadaísmo y el mundo de la nueva ola a fines de la década de los sesenta. Mientras que a los nadaístas se les ha atribuido el mérito de inyectar un mínimo de conciencia social en la escena de la nueva ola, para algunos izquierdistas el acercamiento entre los dos campos fue una prueba más de la retórica vacía del nadaísmo. Como dijo un conocido actor involucrado con el EPL, los nadaístas "se quitaron la careta de falsos rebeldes para unirse al go-gó reaccionario” (Aliocha 1967).

\section{Resistencia de pelo largo}

Comentarios como este ilustran la profunda sospecha con que los militantes de izquierda veían a los nuevaoleros y al movimiento hippie que llegó a Colombia a finales de los años sesenta y pasó a estar estrechamente relacionado con la música rock (Tirado 2014, 174). Aunque su espíritu fundamentalmente rebelde es un tropo recurrente en la historiografía del rock colombiano, las políticas de la cultura temprana del rock and roll estaban decididamente mezcladas. A mediados de la década, la cultura musical de la nueva ola celebró un hedonismo juvenil que se vivía en los bailes de fin de semana en torno a los que giraba la escena. Como dijo Eduardo Arias (2000), "no creo que la música de entonces tuviera una carga más allá del somos jóvenes y queremos divertirnos a nuestra manera” (112).

Las clases media y alta de las que provenían muchos músicos y seguidores de la nueva ola socavaron de manera semejante sus credenciales de oposición. Antes de abrazar el potencial de la música go-gó para canalizar ideas nadaístas, Gonzalo Arango reflexionó: "Lo ye-yé es una generación que se sacude [...] la nada de su vida burguesa. La lástima es que esa sacudida no conduce a nada. Es una generación que ni siquiera se rebela en un sentido creativo” (Osorio 1966, 11). 
Entre las bandas, las posiciones políticas variaban considerablemente. En respuesta a preguntas sobre si apoyaban una revolución en Colombia, uno de los integrantes de Los Flippers afirmó que "de cierto modo nosotros tocamos para olvidarnos de las cuestiones sociales”, mientras que otro indicó que favorecía una revolución al estilo cubano (“Todo lo que hay que saber” 1966). Un grupo de jóvenes músicos de rock and roll, que en 1967 afirmaba estar tras una "revolución” artística, zanjó un contraste explícito entre su propio enfoque y las estrategias de resistencia que favorecía la izquierda, reconociendo que "en música, pintura, canciones y escultura vamos a realizar una 'revolución’ pacífica, sin huelgas, protestas ni nada parecido” (Ayuso 1967, 14).

Si bien había una brecha evidente entre los principios que guiaban a la mayoría de los músicos de la canción protesta y los de la nueva ola, los jóvenes involucrados con este último tipo de música abrazaron una forma de protesta generacional a través de la cual aspiraban a desafiar las nociones tradicionales sobre la vestimenta, la longitud del cabello en los hombres, la sexualidad y la música (López 1994, 72-75; Pérez 2007, 55). Aunque estas disputas -libradas en el frente cultural - no pretendían alterar el orden político o económico, los conservadores percibieron claramente una amenaza a su hegemonía. La policía de Bogotá y Medellín tenía la costumbre de detener a los melenudos, que eran conocidos como los principales adeptos de la nueva ola, y raparles la cabeza a la fuerza (Londoño 2014, 70). En ocasiones, la juventud urbana que hizo de la nueva ola su emblema musical organizaba actos colectivos de resistencia contra la denigración que sufría por parte de la cultura dominante. A finales de 1966, el destacado DJ Jaime Guerra Madrigal organizó una marcha en Medellín para protestar por la difamación de la que eran víctimas los go-gós y ye-yés en esa ciudad; una manifestación similar se llevó a cabo en Bogotá al año siguiente (“Arte y artistas” 1966a; Moannack 1967).

\section{"Sinceridad revolucionaria"}

Puede que los miembros de los partidos políticos de izquierda hayan reconocido la inutilidad de tratar de sacarles partido a las ambiguas inclinaciones opositoras de los fanáticos del rock en aquellos espacios donde ambos interactuaban. Uno de estos fue la Primera Asamblea de la Juventud en Rebelión de 1968, que contó con discursos de los líderes de las alas juveniles de varios partidos y en la que supuestamente se presentarían Los Flippers y Eliana. Después de que la policía prohibiera a los músicos ingresar con sus instrumentos al lugar (el edificio del Capitolio Nacional), el evento casi terminó debido a que "el éxodo de los 
melenudos fue masivo" ("Primera asamblea” 1968, 2). Uno de los escritores de Voz Proletaria criticó a la mayoría de los fanáticos del go-gó que se hallaban entre la multitud, pero sugirió que aquella juventud en la que era posible "apreciar sinceridad revolucionaria, que aplaudía con entusiasmo a Camilo Torres y al Che Guevara”, debía "buscar el verdadero canal revolucionario que moviliza a la juventud a la lucha por la toma del poder bajo la dirección [del] Partido Comunista” ("Primera asamblea" 1968, 2).

Al tomar cierta distancia, podemos distinguir cuatro ejes principales en la crítica de la izquierda a la nueva ola y los hippies. Por un lado, como menciona Giraldo (1997, 15), las raíces extranjeras del rock permitieron que los radicales lo descartaran como síntoma del imperialismo estadounidense. En segundo lugar y siguiendo de nuevo a Giraldo, la mayoría de los hippies se distanciaron de los grupos comunistas en lo relativo a sus posiciones en torno a la insurrección armada. La cobertura de prensa sobre el movimiento insinuó que la juventud hippie del momento pertenecía a "las nuevas generaciones que han resuelto cambiar la piedra y las bombas ‘Molotov’ por una guitarra” (Hurtado 1971, 1B). En tercer lugar, la declaración de la Primera Asamblea de la Juventud en Rebelión, que priorizaba "la lucha por la toma del poder", es evidencia del conflicto elemental entre el enfoque marxista de la lucha de clases y la "atmósfera de resistencia y de crítica a los valores imperantes” en el movimiento hippie (López 1994, 73-74). Por último, la sugerencia de que el Partido Comunista era la mejor ruta para trabajar en favor de un cambio revolucionario refleja los esfuerzos de las organizaciones de izquierda por monopolizar las prácticas de oposición. Si bien el fenómeno de la canción protesta había permanecido vinculado a la esfera de influencia del PCC hasta aproximadamente 1970, en 1971 la prensa identificó los conciertos de rock y los festivales del Coco de Oro como sitios clave para la canción protesta. Dada la tendencia de los activistas a seguir el dogma de sus organizaciones rígidamente, no es sorprendente que menospreciaran la música de protesta producida fuera de su jurisdicción.

\section{Movimientos contraculturales a finales de la década de los sesenta}

A pesar de las brechas ideológicas que existían entre las tres corrientes contraculturales, a partir de 1967 las figuras prominentes del movimiento nadaísta, la escena de la nueva ola y la canción protesta se entremezclaron con frecuencia. 
La relación entre el nadaísmo y la nueva ola fue particularmente fructífera durante este periodo. Al encontrar en la fase go-gó de la nueva ola un aliado en su resistencia a las costumbres tradicionales, los poetas nadaístas se esforzaron por dejar su huella en la escena musical popular. Lo más destacado del Festival de Vanguardia, organizado en 1967 por nadaístas en Cali, fue un concierto que reunió a los “principales artistas go-gó” del país (Morris 1967, 14).

Poco después de ese evento, Gonzalo Arango (1967b) publicó un largo ensayo ensalzando las cualidades revolucionarias del go-gó, destacando sus afinidades con el nadaísmo y anhelando el "nuevo rumbo" hacia el que los escritores nadaístas encaminarían el movimiento go-gó "con sus canciones de protesta y sus baladas de amor” (13). A principios de la década de los setenta, la estima mutua entre los rockeros hippies y los nadaístas se hizo patente. En 1970 la policía arrestó al poeta nadaísta Jotamario Arbeláez junto con “cincuenta hippies” afuera del local donde la banda de rock estadounidense Hope se preparaba para ofrecer un concierto -que terminó siendo prohibido por la Sección de Prevención y Control de Narcóticos de la ciudad de Bogotá (“50 detenidos” 1970)—.

El vínculo entre el nadaísmo y la nueva ola fue más allá de una convivencia intermitente y entró en el ámbito de la colaboración musical. La cantante de nueva ola Eliana grabó dos álbumes de canciones escritas por nadaístas - con títulos como Canciones de la nada (1967) - que aludían al movimiento de forma explícita (Mesa 1967). En relación con esta obra, Gonzalo Arango escribió que “funde dos generaciones: el nadaísmo y el go-gó, la poesía y la música”.

Otra asociación artística clave fue la que formaron Arango y la banda de rock de Medellín Los Yetis. En 1967 Arango publicó un extenso reportaje sobre la banda, en el que elogió a la generación go-gó por actuar como contrapunto a los medios violentos a través de los cuales muchos en Colombia habían resuelto lidiar con sus frustraciones: "Toda esa furia en estado salvaje que se expresaba en una violencia sin objeto, se encarnó en el espíritu go-gó [...] esa generación no expresó su protesta por las armas, sino por el arte” (Arango 1967a, 66). En un segmento novelado al final de la pieza, Arango preguntó a Los Yetis por qué no escribían canciones de protesta. El integrante de la banda Juan Nicolás Estela respondió que, si Arango escribía una para ellos, la grabarían. La canción resultante fue Llegaron los peluqueros (Arango 1967a). Según el cantante de Los Yetis Juancho López, el texto de la canción — que incluía la línea "Mueran los peluqueros, vivan las melenas, la revolución”- expresaba una de las demandas más urgentes de los nuevaoleros masculinos: el derecho a dejarse crecer la melena.

3 Esta cita proviene de lo que parece ser un manuscrito mecanografiado por Arango acerca de la grabación (Nadaísta 2013). 
Por divertida que pueda parecer hoy esta postura, recordemos que en aquel entonces la cabellera de los hombres constituía un sitio clave para el control y la resistencia social. De hecho, Llegaron los peluqueros fue vetada en la radio por el Ministerio de Comunicaciones (Londoño 2014, 80).

Los discursos de resistencia asociados con la nueva ola y el nadaísmo se superpusieron claramente de tal forma que, a la larga, los separaron de la canción protesta. Aun así, los valores sociales progresistas defendidos dentro de los dos primeros compartían, cuando menos, afinidades superficiales con las ideas de izquierda; además existían vínculos importantes entre la comunidad del rock and roll, los nadaístas y el mundo del activismo de izquierda. Los miembros de Los Young Beats, una banda formada en 1966 en Bogotá, tenían vínculos con artistas de izquierda, ensayaban en el Instituto Cultural Colombo-Soviético y transmitían mensajes políticos en sus canciones (Moreno 2003). A fines de la década de los sesenta, un número creciente de estrellas de la nueva ola se sintió atraído por el encanto contestatario de la canción protesta. En 1967 se publicó un perfil detallado del ídolo de la nueva ola Óscar Golden que gravitó en torno a su apoyo al género de canción protesta (“Que no censuren” 1967). Al menos un nuevaolero simpatizó lo suficiente con la retórica comunista que sustenta la canción protesta como para unirse a las filas de la guerrilla. En 1967 el baterista de Los Speakers aparentemente participó en el secuestro de un vuelo doméstico por parte del ELN en protesta por la expulsión de Cuba de la Organización de Estados Americanos (Behar 1985, 112). Los relatos del Festival de la Vida de 1970 - uno de los primeros grandes festivales de rock celebrados en Bogotá- proporcionan un indicador de las orientaciones políticas del movimiento hippie a medida que alcanzaba su cenit: los artistas cantaron sobre el "Che” Guevara y Fidel Castro, así como sobre las huelgas obreras (Levy 1970).

También hubo instancias en que los partidarios de la canción protesta abrieron espacios para los protagonistas de la nueva ola y el nadaísmo. En 1966 la JUCO invitó a grupos de go-gó a actuar durante una semana cultural y, al año siguiente, Los Young Beats actuaron en el nido de la canción protesta, la Casa de la Cultura (Moreno 2003). Reconociendo el mérito de los valores de oposición que los nadaístas expresaban en su producción literaria, los músicos involucrados con el CNCP musicalizaron algunos de sus textos. Entre los poemas nadaístas que Juan Sebastián musicalizó estaba Tomás el Contento, de Gonzalo Arango, que relata la historia de un joven reclutado para “defender a Wall Street en el Vietnam” (Gac y Valencia 1970, 104-105).

La producción musical descrita hasta ahora fue resultado de la inspiración mutua y las colaboraciones entre artistas fuertemente identificados con el nadaísmo, la nueva ola o la canción protesta. Sin embargo, ciertos artistas 
individuales también llegaron a personificar los acercamientos entre estas corrientes. En 1966 Pablus Gallinazo obtuvo el primer premio del Concurso Nadaísta de Novela por su libro La pequeña hermana y se convirtió en una figura clave del movimiento (Pedersen 1971, 363). Ese mismo año, se consolidó como un actor importante en la música de la nueva ola con su composición Boca de chicle, que Óscar Golden convirtió en un éxito (“Arte y artistas” 1966b) ${ }^{4}$.

Gallinazo también estuvo involucrado en el periodo de gestación del CNCP por esta época y demostró sus credenciales comunistas actuando en el cincuentenario de la Revolución rusa. En una línea similar, la cantante Eliana se movió con facilidad entre las tres corrientes: actuó en el Festival Nadaísta de Arte de Vanguardia en 1967 y grabó canciones nadaístas, lanzó álbumes en conjunto con la industria musical que promovía la nueva ola y fue una participante habitual en los eventos del CNCP.

\section{La canción protesta se vuelve comercial}

Mientras los militantes de izquierda disputaban las formas de resistencia que el nadaísmo, la nueva ola y los hippies privilegiaban, algunos partidarios de la canción protesta abrieron campo a otras manifestaciones contraculturales. Los nadaístas, por su parte, rechazaron cualquier tipo de dogmatismo político, incluido el que emanaba de las diversas facciones comunistas — si bien es cierto que algunos estaban de acuerdo con los ideales de izquierda-. Varios nadaístas destacados se sintieron atraídos por las energías rebeldes de la nueva ola y el rock hippie. Y aun cuando ciertos segmentos de los dos últimos movimientos fueron cimentados en torno a un hedonismo y una devoción por la paz, muchos de los jóvenes involucrados en ellos simpatizaron con las ideas políticas que promovían los activistas del movimiento de la canción protesta.

Como espero que haya podido apreciarse en el resumen anterior, pese a los límites discursivos que los ideólogos de estos movimientos contraculturales pudieron haber establecido en torno de ellos, en la práctica dichos límites eran bastante fluidos para los jóvenes urbanos. Estos demostraban tener una variedad

4 Gonzalo Arango describió la canción, que parece expresar un anhelo de sensualidad, como "al mismo tiempo un poema nadaísta y un ritmo 'go-gó"” (Arango 1967a, 12). Es importante señalar que los jóvenes de esa época interpretaban las alusiones al erotismo como formas de resistencia a la cultura que muchas personas con las que hablé veían como puritana. Un hombre de mediana edad a quien conocí en un concierto de Pablus Gallinazo en 2013 me dijo que Boca de chicle era contestataria precisamente por sus connotaciones sexuales. 
de intereses políticos, así como una amplia gama de preferencias artísticas, y entraban y salían de diversos espacios contraculturales. A su vez, sus lealtades hacia músicos o conjuntos particulares - o, en el caso de los propios artistas, a contextos particulares de interpretación y producción- también cambiaron en forma semejante. En la sección final de este artículo, demuestro cómo este entorno dinámico allanó el camino para que la canción protesta llegara más allá de su audiencia original y se convirtiera en un producto cultural de masas. Una breve mirada a las experiencias de dos grupos de jóvenes ayudará a ilustrar esta transición.

Al tiempo que se convertían en unos de los intérpretes más activos del CNCP durante sus años de mayor actividad (1969-1971), los dúos Norman y Darío y Ana y Jaime también echaban raíces en el mundo de la nueva ola. Norman Smith e Iván Darío López habían sido miembros de Los Yetis antes de mudarse a Bogotá alrededor de 1969. Una vez en la capital, el dúo Norman y Darío actuó con frecuencia en las peñas que se celebraban en la Casa de la Cultura. Allí establecieron una relación con el poeta y militante de izquierda Nelson Osorio Marín y compusieron música para varios de sus textos. Los recuerdos que Smith guarda de ese periodo son un testimonio del abigarrado ambiente político dentro del cual él y López crearon su propio estilo de canción protesta:

Vivíamos en Bogotá y, ya sabes, las tendencias de izquierda, porque estábamos molestos y queríamos hablar de eso y ser como todos los demás revolucionarios [...] Supongo que fue bastante político. Quiero decir, o eras de la oligarquía o no lo eras [...] Otras personas eran mucho más intensas y tenían sentimientos y pensamientos más estructurados sobre por qué estaban haciendo lo que estaban haciendo [...] Nosotros estábamos como flotando con todas estas personas diferentes, pensando lo mismo pero sin adherirnos a ningún llamado o bandera en particular. (Norman Smith, entrevista, febrero de 2015)

El dúo Norman y Darío utilizó sus contactos con Los Yetis para establecer conexiones con los medios de comunicación, trabajando de cerca con Alfonso Lizarazo y actuando en Estudio 15 -su programa de televisión orientado hacia la nueva ola-. En su breve carrera como dúo, Norman y Darío grabaron con CBS un mini-LP y un LP. La copia física que obtuve del mini-LP confirma que fue apreciada por intelectuales de izquierda: la presencia de sellos en la portada del disco indica que perteneció al Club Máximo Gorki. Puesto que lleva el nombre del pionero del realismo socialista, cuyo centro de operaciones se encontraba en el Instituto Cultural Colombo-Soviético y que anunciaba sus actividades en $\mathrm{Voz}$ Proletaria, es probable que este grupo tuviera algún tipo de relación con el PCC (“Culturales” 1970). El LP, titulado Las primeras protestas, parece encontrarse 
en sintonía con las producciones de la nueva ola de esa época. Lizarazo fue el director artístico de la grabación y los arreglos estuvieron a cargo de Harold. Las notas en la contraportada del disco describen su contenido como "un desafío al tradicionalismo".

El dúo conformado por los hermanos Ana y Jaime se involucró con la canción protesta al mismo tiempo que lo hicieron Norman y Darío. Al igual que Smith y López, los hermanos Valencia no estaban asociados a ningún partido político, pero se sintieron atraídos por el clima de oposición que se vivía en el CNCP:

Allí nosotros conocimos canciones sociales, como que denunciaban algo [...] Éramos muy niños, pero nos gustaba lo que contábamos, nos gustaban las canciones que ellos cantaban, nos parecían bonitas. Nos parecía que funcionaban o que la gente entendía el mensaje que se quería dar. (Ana Valencia, entrevista, marzo de 2015)

Los asistentes a las peñas quedaron encantados con el dúo, por lo que les regalaron discos y les enseñaron temas del repertorio latinoamericano de canción protesta. Nelson Osorio pidió a los jóvenes que musicalizaran sus textos y algunas de sus canciones más conocidas fueron el resultado de sus colaboraciones con él. Sin embargo, mientras Ana y Jaime se integraban al movimiento en el CNCP, Alfonso Lizarazo los alistaba para el mercado comercial, invitándolos a actuar en sus programas de talentos y preparándolos para grabar su primer LP.

Inicialmente, la comunidad del CNCP acogió a Norman y Darío y a Ana y Jaime, quienes se presentaron en el Primer Festival de la Canción Protesta, junto a los principales organizadores del CNCP. Cuando los dúos no fueron incluidos en el cartel inicial del primer Festival de la Canción Protesta Coco de Oro en 1970, sus colegas del CNCP escribieron una carta a los organizadores condenando su exclusión. Alejandro Gómez afirmó que el festival

no podía ser la expresión de lo que es la Canción Protesta. Se escogieron a los cantantes que nunca han tenido que ver con este movimiento e improvisadamente trataron de aprenderse varias canciones protesta, lo que para ellos era un género desconocido. (“Canción protesta” 1970)

Ana y Jaime y Norman y Darío — el último de los cuales fue calificado por Gómez como "uno de los mejores, si no el mejor dueto juvenil que hay en Colombia”- terminaron presentándose en el festival. Sin embargo, al igual que sucedió durante la segunda edición al año siguiente, el montaje del primer Coco de Oro en la meca turística de San Andrés fue inusitado. Organizado por el cantante de nueva ola Leonardo Álvarez, el evento tuvo como objetivo recaudar fondos para la construcción de una capilla católica en la isla y el jurado incluyó a un sacerdote local, junto con el superintendente de San Andrés, Pedro López 
Michelsen, el escritor izquierdista Jaime Mejía Duque y Gonzalo Arango —otrora un crítico intrépido de la Iglesia (Samuel 1970)—.

Norman y Darío se separaron en 1971 cuando Smith se mudó a los Estados Unidos. Ana y Jaime, sin embargo, se continuaron reorientando hacia la infraestructura mediática que había sido creada para la nueva ola, al tiempo que conservaron su rótulo como exponentes de la canción protesta. Y así fue como llegaron a ser concursantes en el Festival Coco de Oro de 1971. Como se describe en la introducción a este artículo, el cartel del festival también incluyó a músicos de la nueva ola que tenían poca relación con la canción protesta. En contraste con los esfuerzos del CNCP por atraer a las clases trabajadoras reduciendo el costo de entrada a sus peñas, la tarifa de ingreso al Coco de Oro era inasequible para esa población. El festival giró en torno a un concurso de canciones que un dramaturgo de izquierda, el fundador del nadaísmo, empresarios locales, figuras de los principales medios de comunicación y funcionarios del Gobierno fueron invitados a juzgar. Muchos observadores cuestionaron la presencia de emisarios del "establecimiento” entre el jurado y existió el temor de que la composición de este llevara a la censura. Sin embargo, algunos de los jueces tenían nociones alineadas con las de los activistas del CNCP sobre lo que debería ser una canción de protesta y las canciones del repertorio popular de este género finalmente fueron premiadas.

En mi opinión, los numerosos aspectos contradictorios de los festivales del Coco de Oro y las controversias que engendraron fueron manifestaciones de la colisión entre discursos de resistencia contrapuestos. A medida que surgía una contracultura juvenil más generalizada que se basó en la combinación de diferentes corrientes, los actores ajenos a la comunidad del CNCP comenzaron a apostarle a la canción protesta. En tanto que el género empezó a ser apropiado más allá de su contexto original, las concepciones acerca de la canción protesta empezaron a apartarse de la visión cultivada en el CNCP.

Cabe destacar que aquellos artistas que habían estado involucrados con el CNCP, pero que también participaron en los festivales del Coco de Oro, transmisiones de radio y televisión y lanzamientos de álbumes con compañías discográficas, perdieron el favor de las voces más definidas políticamente en el movimiento de base. En 1973 Alejandro Gómez dijo sobre Ana y Jaime que, si bien era loable que el grupo incluyera textos de Nelson Osorio en sus discos, "[m]ás tarde se independizaron [del CNCP] y se comercializaron, desafortunadamente” (Gómez 1973). Resumiendo su opinión sobre artistas como Ana y Jaime y Pablus Gallinazo, lamentó:

Esta es a nuestro modo de ver, una canción protesta entre comillas, comercializada por las empresas disqueras que están interesadas en que esta clase de canciones sea la que más se oiga como canción protesta, mientras 
que las verdaderas canciones comprometidas, las canciones revolucionarias, sí están totalmente olvidadas por tales empresas. (Gómez 1973)

En retrospectiva, las preocupaciones de Gómez no estaban del todo fuera de lugar. Lo que desde entonces ha venido a conocerse en Colombia de modo general como canción protesta es precisamente la versión que él reprobó. En mi opinión, sin embargo, su teoría acerca de una conspiración de las empresas para acabar con la genuina resistencia musical en la canción protesta es demasiado simplista. Esta conjetura desmiente el hecho de que hubo una superposición sustancial entre las canciones de protesta que se escucharon en los medios y las que aparecen en los cancioneros compilados por activistas de izquierda.

\section{Conclusiones}

Situar las expresiones contraculturales y de oposición en los discursos de resistencia en los que adquirieron sentido puede ayudarnos a comprender los itinerarios que la canción protesta recorrió a principios de la década de los setenta. Durante este periodo, los discursos asociados a la canción protesta y la nueva ola - la última de las cuales estaba inspirada en el nadaísmo- coincidieron en algunos nodos clave. Estas pequeñas superposiciones abrieron la posibilidad de que los grupos e instituciones que fomentaban la nueva ola y el rock confluyeran en la esfera de acción del género de canción protesta y tomaran algunas de sus connotaciones originales. La respuesta de los activistas de izquierda a lo que percibieron como la comercialización de la canción protesta -que comunicaba los preceptos centrales del discurso dentro del cual se desarrolló- es testimonio de la naturaleza altamente contingente de la resistencia musical. De hecho, los artistas y las canciones que los discípulos del CNCP desdeñaron cuando se quebraron los vínculos entre estos y el movimiento de base no eran distintos a los que habían aplaudido en las peñas del CNCP. Sin embargo, al ser difundidos en contextos diferentes, estos mismos gestos musicales terminaron siendo incompatibles con la concepción que tenían los comunistas acerca de la direccionalidad de la resistencia.

Enfocarse en los discursos de resistencia también ayuda a eludir la trampa de describir la música contestataria como "música de protesta” o "música de resistencia" - un dilema que proviene de la tendencia, recurrente en algunos escritos musicológicos, a proyectar las características de la resistencia cotidiana 
sobre toda resistencia musical—. Desde una perspectiva más amplia, podemos considerar varias de las acciones aquí descritas (vandalizar una iglesia, escribir poemas nadaístas, bailar con lujuria al ritmo del rock and roll, interpretar canciones de protesta que exaltan a los grupos guerrilleros, hombres que se dejan el pelo largo, campesinos que toman las armas, etc.) como actos potenciales de resistencia que se encuentran en el espectro entre lo cotidiano/individual y lo excepcional/colectivo. En combinación con un análisis detallado, este enfoque también proporciona un marco útil para identificar las espinosas convergencias entre resistencia y poder, tal y como fueron negociadas por diversos actores desde sus respectivas posiciones en el contexto de una sociedad cambiante (por ejemplo, músicos, activistas, periodistas, políticos, fuerza policial, etc.). Para concluir donde comencé, ofrezco el ejemplo de la canción ganadora del Festival coco de Oro de 1971.

A mediados de la década de los sesenta, el cantante y guitarrista Sergio Torres atrajo la atención de la prensa gracias a una larga gira que lo llevó por Europa interpretando música tradicional colombiana. Aun cuando Torres asumió “posiciones revolucionarias” durante su estadía en Europa, en Colombia no estuvo involucrado en el movimiento de la canción protesta (Torres 2018). Se trata, pues, de uno de los tantos músicos que participaron en los concursos de canción protesta del Coco de Oro, pese a no haber estado afiliados previamente al género, situación que molestó a periodistas, jueces y activistas del CNCP. En lo que a las canciones de protesta respecta, sin embargo, el tema de Torres No trabajo más se ajusta a los modelos establecidos por los músicos activistas que impulsaron el género. De hecho, los maoístas que en 1971 publicaron un cancionero de protesta titulado Guitarra y fusil la consideraron digna de ser incluida, y uno puede fácilmente imaginar a los jóvenes militantes enardeciendo una multitud con su estribillo simple y pegadizo.

Si bien el mismo Torres grabó la canción, su triunfo en el festival inspiró a la orquesta tropical venezolana Los Melódicos, que era bastante popular en Colombia, a producir su propia versión en ritmo de cumbia. Curiosamente, la animada versión omite uno de los versos originales:

Trabajo en las petroleras

Con ese calor minero

Las empresas extranjeras

Se llevan nuestro dinero

Es tentador interpretar esta edición como un reconocimiento tácito de que este fragmento de la canción, que denuncia la explotación neocolonial de los recursos naturales de Latinoamérica, era la más propensa a ser leída como 
subversiva. De hecho, a pesar de haber sido aprobada por políticos y otros miembros del jurado del Coco de Oro que "no eran verdaderamente revolucionarios", los medios de comunicación y las entidades gubernamentales parecen haber intentado aplastar la difusión de No trabajo más. Como informó Torres en una entrevista, "esa canción tuvo un veto por una cadena radial [...] y también hubo rumores que había sido prohibida por el Ministerio de Comunicación, porque en Barrancabermeja la cogieron como himno, y porque la consideraban comunista” (Guzmán ca. 1972).

Si aquello que Torres sugiere es verídico, la posibilidad de que los obreros en los campos petroleros de Barrancabermeja reclamaran por sus condiciones laborales puede haber llevado a los funcionarios del Gobierno a ejercer su poder para limitar la difusión de la canción. En contraste con un músico de clase media que entona protestas abstractas en el escenario de un festival, tal suceso puede haber presagiado una forma de resistencia colectiva pública con el potencial para producir cambios sociales.

\section{Referencias}

\section{Fuentes primarias}

“50 detenidos al prohibir policía concierto 'rock”. 1970. El Tiempo, 11 de octubre, 7.

Aliocha. 1966. “Poesía y revolución”. Cromos, 12 de diciembre, 84.

—. 1967. "Fausto y la tragedia del humanismo". Cromos, 14 de agosto.

—. 1968. "Última página”. Cromos, 15 de abril.

Arango, Gonzalo. 1967a. “Cara a cara con Los Yetis”. Cromos, 28 de agosto, 62-65.

—. 1967b. “Qué diablos es el go-gó?”. Cromos, 7 de agosto, 10-13.

Arias, Eduardo. 2000. "El rock colombiano: el proceso de surgir y de caer varias veces”. Gaceta $47,112-117$.

“Arte y artistas”. 1966a. El Espectador, 9 de diciembre, B4.

“Arte y artistas”. 1966b. El Espectador, 14 de diciembre, B4.

Ayuso, Miguel. 1967. “'Revolución’ artística inician 15 conjuntos”. El Tiempo, 27 de abril, 14.

“Canción protesta es cantar los problemas que vivimos”. 1970. Voz Proletaria, Suplemento Juventud, 13 de agosto. 
“Canciones de protesta y esperanza”. 1968. Voz Proletaria, 25 de abril, 2.

“Contenido”. 1971. El Tiempo, 16 de julio, 18.

“Culturales”. 1970. Voz Proletaria, 12 de febrero, 7.

“Del nadaísmo al oficialismo”. 1968. Voz Proletaria, 14 de noviembre, 5.

Dilday, Jessica. 2014. “IASPM-US Interview Series: Roll with it, by Matt Sakakeeny”. International Association for the Study of Popular Music - US Branch, 18 de agosto. http://iaspmus.net/iaspm-us-interview-series-roll-with-it-by-matt-sakakeeny/

Emanuelsson, Dick. 2014. "Falleció en Bogotá el compositor de ‘Cuba sí, yanquis no!”. 45 revoluciones por minuto, 10 de septiembre. http://45-rpm.net/blog/2014/09/10/fallecio-enbogota-el-compositor-de-cuba-si-yanquis-no/

“Entrega del ‘Coco de Oro””. 1971. El Tiempo, 21 de julio, 11.

Escobar, Eduardo. 1976. “Gonzalo Arango. El nadaísmo. Historia de una pasión”. Gaceta 7, 7-10.

Gómez, Alejandro. 1973. “Con Alejandro Gómez”. Boletín Música Casa de las Américas 42.

“Gran festival de la canción protesta”. 1970. Voz Proletaria, 24 de septiembre, 7.

“Grandioso acto rendido a la U.R.S.S.”. 1967. Voz Proletaria, 16 de noviembre, 10-11.

Guzmán, Alberto. Ca. 1972. "Sergio Torres: la nariz que canta”. Recorte de prensa, s. d.

Hurtado de Paz, Amparo. 1971. “3 días de música en Medellín”. El Espectador, 18 de junio, 1B.

II Festival de la Canción Protesta 'Coco de Oro’ San Andrés. 1971. Programa de concierto. San Andrés: Junta directiva del festival.

Levy, Alegre. 1970. “El festival de la vida”. El Tiempo, 28 de junio, 16.

—. 1971. "Cantos y desencantos del Festival Protesta”. El Tiempo, 23 de julio, 17.

“Lo que dejó la tempestad”. 1979. Alternativa 207, 9 de abril, 25-27.

“Los sábados obreros”. 1971. Voz Proletaria, 18 de febrero, 6.

Mesa, Elkin. 1967. "En tono confidencial”. Cromos, 3 de julio.

Moannack, Gloria. 1967. “La marcha ye-yé de la 'nueva civilización””. El Tiempo, 12 de febrero, 15.

Moreno, David. 2003. “Rock y política de izquierda en Bogotá, parte 2: Chapinero a go-go”. Estudiocaos.com, 15 de mayo. http://www.lasillaelectrica.com/articulos_rockizquierda02. htm

Morris de Flórez, Athala. 1967. "Breves del festival”. El Tiempo, 14 de junio, 9.

Nadaísta. 2013. “Gonzalo sobre Eliana” (imagen digital). Flickr, 2 de agosto. https://www. flickr.com/photos/89185403@N05/9424456777/

“Noticiero cultural”. 1968. El Tiempo, 22 de febrero, 14. 
“Organizan festival de la canción protesta”. 1970. Voz Proletaria, 10 de septiembre, 6.

Osorio, Sonia. 1966. "El sacudido mundo de los ye-yé”. Cromos, 12 de diciembre, 11.

“Primera asamblea de la ‘juventud en rebelión””. 1968. Voz Proletaria, 3 de octubre, 2.

“Programación de la Casa de la Cultura”. 1970. Voz Proletaria, 15 de enero, 6.

“Que no censuren las canciones de protesta pide Golden”. 1967. Cromos, 10 de julio, 22-27.

Samuel. 1970. “Festival de protesta se inicia hoy en San Andrés”. El Tiempo, 3 de julio, 7.

—. 1971a. “Animación y protestas en festival de San Andrés”. El Tiempo, 18 de julio, 9.

—. 1971b. "Festival de San Andrés más canción que protesta”. El Tiempo, 20 de agosto, 19.

“Todo lo que hay que saber sobre Los Flippers”. 1966. Cromos, 28 de noviembre.

Torres del Castillo, Iván. 2018. "Mini biografía artística de Sergio Torres Molina”. Manuscrito inédito.

\section{Fuentes secundarias}

Abu-Lughod, Lila. 1990. "The romance of resistance: tracing transformations of power through Bedouin women”. American Ethnologist 17 (1): 41-55. http://www.jstor.org/stable/645251

Arango, Gonzalo. 2013. Primer manifiesto nadaísta y otros textos. Compilado por Philippe Ollé-Laprune. Ciudad de México: Vanilla Planifolia.

Archila Neira, Mauricio. 2003. Idas y venidas, vueltas y revueltas: protestas sociales en Colombia, 1958-1990. Bogotá: ICANH; Cinep.

Attfield, Sarah. 2017. “My manor's ill': how underground music told the real story of the UK riots”. En Youth culture and social change: making a difference by making a noise, editado por Keith Gildart, Anna Gough-Yates, Sian Lincoln, Bill Osgerby, Lucy Robinson, John Street, Peter Webb y Matthew Worley, 79-99. Londres: Palgrave Macmillan.

Behar, Olga. 1985. Las guerras de la paz. Bogotá: Planeta.

Beltrán, William M. 2002. "Del dogmatismo católico al dogmatismo de izquierda. El ambiente político en la Universidad Nacional en los 60s y 70s”. Revista Colombiana de Sociología 7 (2): 155-178. https://revistas.unal.edu.co/index.php/recs/article/view/11166

Díaz, Clara. 1994. Sobre la guitarra, la voz: una historia de la nueva trova cubana. La Habana: Letras Cubanas.

Flores, Arturo C. 1994. “Gustavo Gac o los sueños de un viaje inconcluso”. Revista Chilena de Literatura 45: 143-149. https://www.jstor.org/stable/40356783

Foucault, Michel. 1978. The history of sexuality. Nueva York: Pantheon Books.

Gac Artigas, Gustavo y Perla Valencia Moncada. 1970. Antología de canción protesta. Bogotá: Colombia Nueva. 
Giraldo Ramírez, Jorge. 1997. “Rock e ideología: exclusión, simulación e identidad”. En $M e$ dellín en vivo: la historia del rock, editado por Omar Urán, 12-28. Medellín: Ministerio de Educación Nacional.

Gledhill, John. 2012. "Introduction: a case for rethinking resistance”. En New approaches to resistance in Brazil and Mexico, editado por John Gledhill y Patience A. Schell, 1-20. Durham, NC: Duke University Press.

Hollander, Jocelyn A. y Rachel L. Einwohner. 2004. “Conceptualizing resistance”. Sociological Forum 19 (4): 533-554. https://doi.org/10.1007/s11206-004-0694-5

Johansson, Anna y Stellan Vinthagen. 2020. Conceptualizing 'everyday resistance': a transdisciplinary approach. Nueva York: Routledge.

Lagos, Ramiro. 1977. “De la vanguardia al nadaísmo”. Anales de Literatura Hispanoamericana 6: 97-107. https://revistas.ucm.es/index.php/ALHI/article/view/ALHI7777110097A

Laine, Sofia, Leena Suurpää y Afifa Ltifi. 2018. "Respectful resistance: young musicians and the unfinished revolution in Tunisia". En What politics? Youth and political engagement in Africa, editado por Elina Oinas, Henri Onodera y Leena Suurpää, 58-74. Leiden: Brill.

Laing, Dave. 2003. "Resistance and Protest". En Continuum encyclopedia of popular music of the world, vol. 1, Media, industry, and society, editado por John Shepherd, David Horn, Dave Laing, Paul Oliver y Peter Wicke, 345-346. Londres; Nueva York: Continuum.

Loaiza Cano, Gilberto. 2004. "Los intelectuales y la historia política en Colombia”. En La historia política hoy: sus métodos y las ciencias sociales, editado por César Augusto Ayala Diago, 56-94. Bogotá: Universidad Nacional de Colombia, Facultad de Ciencias Humanas, Departamento de Historia.

Londoño, Diego. 2014. Los Yetis, “una bomba atómica a go go": la historia de los abuelos de nuestro rock. Medellín: Pulso \& Letra Editores.

López de la Roche, Fabio. 1994. Izquierdas y cultura política: ¿oposición alternativa? Bogotá: Cinep.

Manzano, Valeria. 2010. "Ha llegado la 'nueva ola’: música, consumo y juventud en la Argentina, 1956-1966”. En Los 60' de otra manera: vida cotidiana, género y sexualidades en la Argentina, editado por Isabella Cosse, Karina Felitti y Valeria Manzano, 19-60. Buenos Aires: Prometeo.

McDonald, David A. 2013. My voice is my weapon: music, nationalism, and the poetics of Palestinian resistance. Durham, NC: Duke University Press.

Moreno Almeida, Cristina. 2017. Rap beyond resistance: staging power in contemporary Morocco. Cham: Springer International.

Pedersen, Carl Erol. 1971. "Main trends in the contemporary Colombian novel, 1953-1967". Tesis doctoral en Español, The Graduate School, University of Southern California, Los Ángeles.

Pérez, Umberto. 2007. Bogotá, epicentro del rock colombiano entre 1957 y 1975: una manifestación social, cultural, nacional y juvenil. Bogotá: Alcaldía Mayor de Bogotá. 
Riaño, Félix (pseud. Félix Sant-Jordi). 2014. Memoria del rock colombiano. Edición Kindle $1.01 \mathrm{ed}$.

Romero, Armando. 1988. El nadaísmo colombiano o la búsqueda de una vanguardia perdida. Bogotá: Tercer Mundo.

Sakakeeny, Matt. 2013. Roll with it: brass bands in the streets of New Orleans. Con imágenes de Willie Birch. Durham, NC: Duke University Press.

Scott, James C. 1985. Weapons of the weak: everyday forms of peasant resistance. New Haven: Yale University Press.

Street, John. 2012. Music and politics. Cambridge, RU: Polity Press.

Tirado Mejía, Álvaro. 2014. Los años sesenta: una revolución en la cultura. Bogotá: Debate.

Tranmer, Jeremy. 2019. “OK Computer: a sign of the political and ideological times?”. Revue LISA / LISA E-Journal 17 (1). https://doi.org/10.4000/lisa.10621

Vinthagen, Stellan y Anna Johansson. 2013. “'Everyday resistance’: exploration of a concept and its theories”. Resistance Studies Magazine 1. https://doi.org/10.1177/0896920514524604 